\section{Fraktionelles exhaliertes Stickoxid ist guter Prädiktor des Steroid- Ansprechens}

Price DB et al. Fractional exhaled nitric oxide as a predictor of response to inhaled corticosteroids in patients with non-specific respiratory symptoms and insignificant bronchodilatator reversibility: a randomised controlled trial. Lancet Respir Med 2017; DOI 10.1016/S2213-2600(17) 30424-1

Symptome wie Husten oder Kurzatmigkeit sind ein häufiges Problem und können vor allem dann schwer einzuschätzen sein, wenn sie unspezifisch sind. Auch Therapieentscheidungen können in diesen Fällen recht schwierig sein. Häufig werden dann inhalative Steroide verschrieben, jedoch ist das Ansprechen darauf sehr variabel. Ein möglicher Ansatz könnte es sein, das Ansprechen mithilfe des fraktionellen ausgeatmeten Stickoxids vorherzusagen.

Eine internationale Arbeitsgruppe um David Price ging dieser Frage nun in einer doppelblinden, randomisierten und placebokontrollierten Studie nach. Eingeschlossen wurden bis dahin undiagnostizierte Patienten mit Husten, Giemen oder Dyspnoe und weniger als $20 \% \mathrm{Re}$ versibilität auf Bronchodilatoren. Innerhalb der ersten zwei Wochen fanden die Eingangsuntersuchungen statt, anschließend wurden die Patienten randomisiert auf zwei Gruppen verteilt. Sie erhielten über einen Zeitraum von 4 Wochen entweder extrafeine inhalative Kortikosteroide (QVAR $80 \mu \mathrm{g}$ zweimal täglich 2 Hub, äquivalent zu $800 \mu \mathrm{g}$ Beclomethason Dipropionat täglich) oder Placebo. Die Randomisierung wurde anhand des exhalierten Stickoxids in der Eingangsuntersuchung stratifiziert: normal $(\leq 25$ ppb), intermediär (>25 bis<40ppb) und hoch ( $\geq 40 \mathrm{ppb}$ ). Primärer Endpunkt war die Veränderung im mittleren Punktwert des Asthma Control Questionnaire (ACQ7).

294 Patienten nahmen an der Studie teil, davon 148 in der Verum- und 146 in der Placebogruppe. In die Analyse gingen letztlich 214 Patienten (114 inhalatives
Kortikosteroid und 100 Placebo) ein im Durchschnittsalter von 50,0 bzw. 47,7 Jahren. Es fand sich in der Behandlungsgruppe eine signifikante Assoziation zwischen dem fraktionellen exhalierten Stickoxid in der Eingangsuntersuchung und dem Therapieansprechen. Dabei zeigte sich, dass das Ausmaß des Ansprechens vom Stickoxid-Wert abhing. Für jede Zunahme um 10 ppb stieg die Veränderung im Asthma Control Questionnaire in der Behandlungsgruppe im Vergleich zur Placebogruppe um 0,071 an. Die häufigsten Nebenwirkungen bestanden in einer Nasopharyngitis (12\% Verum vs. 9\% Placebo), Infektionen (17\% vs. $14 \%$ ) und respiratorischen, thorakalen oder mediastinalen Beschwerden ( $9 \%$ vs. $12 \%)$.

FAZIT

Die Messung des fraktionellen exhalierten Stickoxids ist ein einfaches nicht invasives Instrument für die klinische Praxis, um bei Patienten mit unspezifischen respiratorischen Symptomen das Therapieansprechen auf inhalative Kortikosteroide vorherzusagen, so die Autoren. Zukünftig müsse nach ihrer Ansicht auch die Rolle der Methode bei anderen Atemwegserkrankungen wie beispielsweise der COPD untersucht werden.

Dr. med. Johannes Weiß, Bad Kissingen 